\title{
THE RENAISSANCE OF FRICTION: FROM EMPIRISM TO PHYSICS - AT THE NANOSCALE ${ }^{1}$
}

\author{
ERIO TOSATTI (*)
}

SunTO. - L'attrito e la sua scienza detengono diversi records. Uno è la longevità : da che esiste, l'umanità ha dovuto farci i conti. Ancora oggi, eliminare (o accrescere) l'attrito restano obiettivi tecnologici e pratici di di enorme importanza - si legge per esempio che non meno del $5 \%$ dell'energia prodotta ogni giorno viene sprecato e degradato in calore nocivo. Un secondo record è che malgrado l'intervento di grandi scienziati come Leonardo, che iniziò a mettere la fisica dell'attrito su basi scientifiche già cinque secoli $\mathrm{fa}$, manca a tutt'oggi una formulazione teorica degna di questo nome. Teorici come noi si limitano per lo più a quello che P.W. Anderson in un ambito diverso definì scherzosamente "the indignity of numerical simulations". Tuttavia, il progresso scientifico non ha luogo perchè è necessario, ma perchè è possibile. Negli ultimi decenni, a partire da tecniche sperimentali meso- e nanoscopiche che hanno aperto una nuova finestra sulla fisica dell'attrito al livello atomico e molecolare, è iniziato un progresso, sia simulatorio the teorico, di cui parlerò brevemente.

$* * *$

ABSTRACT. - Friction and its science hold more than a record. One is longevity: from its very origins, mankind has had to reckon with it. Still today, reducing (or increasing) sliding friction remain technological and practical objectives of enormous importance - one can read for example that no less than $5 \%$ of all energy produced daily degrades into wasted frictional work. Another record is that despite the involvement of great scientist like Leonardo, who already five centuries ago gave friction its first scientific bases, there is still today no proper theoretical formulation of friction. Theorists like us

(*) Istituto Lombardo Accademia di Scienze e Lettere, Milano. Scuola Internazionale Superiore di Studi Avanzati (SISSA). Abdus Salam International Centre for Theoretical Physics (ICTP), Trieste, Italia. E-mail: tosatti@sissa.it; tosatti@ictp.it

${ }_{1}$ Work supported by ERC Grant No. 320796 MODPHYSFRICT. Collaboration with P.P. Baruselli, A. Benassi, R. Capozza, Y. Crespo, M. Fabrizio, L. Gigli, R. Guerra, A. Laio, D. Mandelli, N. Manini, E. Panizon, F. Pellegrini, S. Prestipino, G.E. Santoro, M. Teruzzi, A. Vanossi, T. Zanca is gratefully acknowledged. 
mostly limit themselves to what P.W.Anderson jokingly defined in a different context "the indignity of numerical simulations". However, progress in science does not take place because it is necessary, but because it is possible. In the last decades, new mesoscopic and nanoscopic experimental techniques opened new windows on frictional phenomena at the atomic and molecular level. Jump-started by the necessity and by the challenge to understand some of that data, theory and simulation progress is moving on along some lines which I will briefly describe.

\section{INTRODUCTION}

Sliding friction between solids plays a central role in diverse systems and phenomena that span a huge range of scales, from the nanometer contacts inherent in nanosystems and biological molecular motors to the geophysical scales characteristic of earthquakes. Because of its enormous practical and technological importance, friction has stimulated progress over the centuries. Historical figures from Leonardo da Vinci onwards brought friction from engineering into the field of physics, with the formulation of time-honored phenomenological frictional laws, often referred to as the Coulomb-Amontons laws, briefly summarized as follows: (i) the frictional force is independent of the apparent area of contact, (ii) the frictional force is proportional to the normal load, (iii) kinetic friction (the force to keep relative motion at constant speed) does not depend on the sliding velocity and is smaller than static friction (the force needed to initiate motion between two contacting bodies at rest). Subsequently, in the light of a mass of empirical data, important attempts were made in the first half of the 20th century toward a microscopic understanding of these laws. In spite of that, the field as a whole has (with notable exceptions) failed to attract adequate interest by the physicist until the last few decades. A lack of microscopic data, and a corresponding lack of theory, contributed to project an unattractive image of sliding friction, a boring subject rather than a challenge.

Three quiet revolutions, of broad nature and unrelated to friction, are changing the state of affairs over the last two or three decades. First, theoretical progress in the general area of complexity provided new tools to tackle nonequilibrium disordered systems with interacting degrees of freedom. Second, and crucial, the developments in nanotechnology extended the study of friction and permitted its analysis at the nanoscale and microscale, now on well-characterized systems and 
surfaces. The invention in 1986 of scanning tip instruments of the atomic force microscope (AFM) family opened nanofriction as a brand new avenue; the surface force apparatus (SFA) led from about the same time to systematic studies of confined mesoscopic systems under shear; from 1988 moreover, other instruments such as the quartz crystal microbalance (QCM) measured the inertial sliding friction of adsorbate submonolayers. Thanks to these and similar methods, a mass of fresh data and information on well-defined systems, surfaces, materials, and physical conditions has accumulated in the last three decades. Third, finally, computer simulations underwent strong progress, thanks to new techniques and codes, helped by the fantastic growth of computer power. The numerical study of frictional models, as well as atomistic molecular dynamics simulations, are now well teste, ready to provide key advances to our physical understanding.

I will of course not be able to address all the progress intervened thanks to these revolutions. The task I give myself is simply to sketch, mainly based on a few case studies developed in our condensed matter theory group in Trieste, a small fraction of the big scene which I am more familiar with. In the spirit of a brief popular presentation, moreover, equations and references are, with few exceptions, generally omitted. The main sources and details can be found, e.g., in review articles, such as N. Manini, et al., Current trends in the physics of nanoscale friction, Advances in Physics X, 2, 569 (2017), and A. Vanossi, et al., Colloquium: "Modeling friction: From nanoscale to mesoscale, Reviews of Modern Physics 85, 529 (2013), and several others cited in there.

\section{Mechanisms of SLiding Friction}

The mutual rubbing of two real-life bodies is generally a messy affair. The sliding contact between solid surfaces may be clean or dirty or lubricated, smooth or rough, gentle and wearless or strong with wear, electronic or structural, etc. Depending on specifics of each case, the main friction mechanisms commonly recognized can be separated, roughly speaking, into:

1. Entanglement of asperities, plastic deformation, wear, irreversible (commonest).

2. Phononic dissipation, wearless, reversible (flat, hard, dry contacts). 
3. Viscous friction (fluid or lubricated interfaces).

4. Viscoelastic dissipation (e.g., car tyres).

5. Electronic friction (metals, just being established), magnetic friction.

6. Vacuum friction (speculative so far).

From the practical and technological viewpoint, the most important ones are obviously 1,3 and 4 . Conversely, the areas where recent physics progress is being made are mostly 2 and 5 , i.e., phononic and electronic friction. Here I shall exemplify their atomistic understanding by means of theory and simulation. Before doing that, I must briefly mention the main nanoscale experiments that made this development possible.

\section{NANO- AND MESO-SCALE FRICTION EXPERIMENTS}

The workhorses of atomistic sliding friction are the nanoscale, tip-based experiments of the Atomic Force Microscope (AFM) family (Fig. 1) and the mesoscale experiments of the Surface Force Apparatus (SFA) family (Fig. 2, left). Less popular, but not less interesting, is the Quartz Crystal Microbalance (QCM), also mesoscopic (Fig. 2, right). In AFM, the friction force felt by a tip which slides in contact with a surface is directly measured by the tip deflection. A tip in dry contact with a surface generally advances by "atomic stick-slip" showing a friction force map with similar detail to a standard topography map.
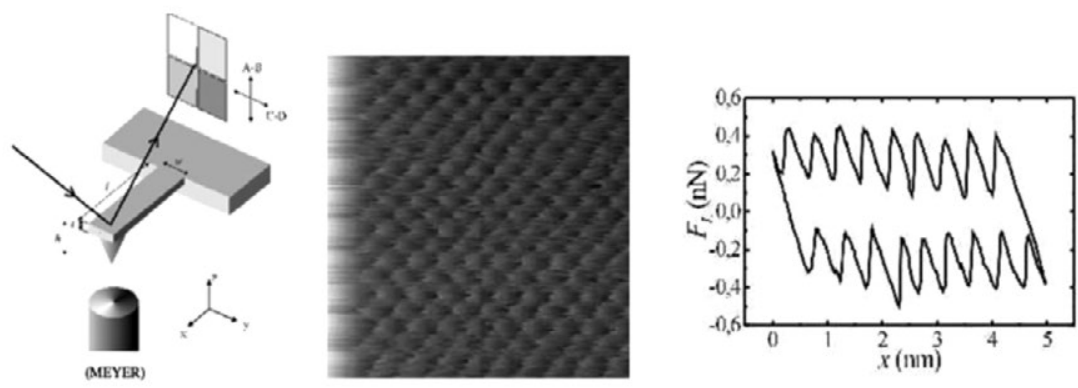

Fig. 1. Left: sketch of AFM for friction. Middle and right: nanofriction force map of a sliding tip on a $\mathrm{NaCl}(100)$ crystal surface measured by AFM (after E. Gnecco et al., J. Phys.: Condens. Matter 13, R619-R642 (2001)). Reproduced by permission of the authors. 

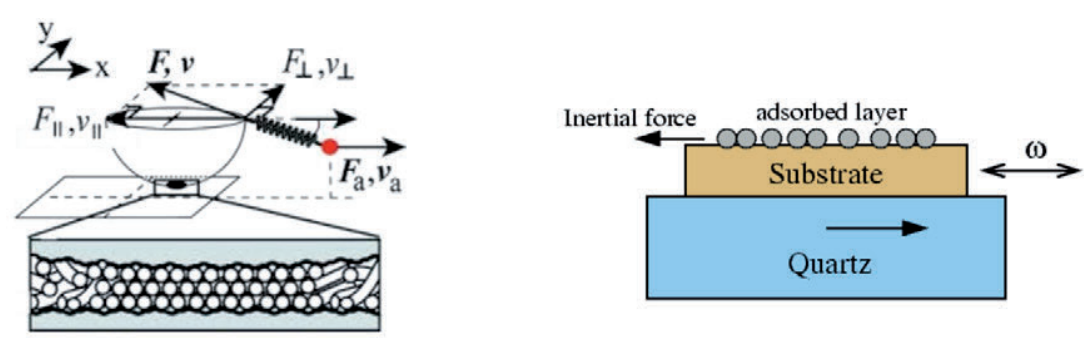

Fig. 2. Mesoscopic friction measured (left) in SFA by shearing a lubricant film squeezed between two very flat crystal surfaces and (right) in QCM by oscillating a $2 D$ solid adsorbed layer and detecting frictional dissipation caused by inertial sliding.

The force-displacement area over a forward-backward scan cycle (Fig. 1) measures the frictional energy of the AFM cycle. In noncontact, conversely, frictional dissipation is measured by the cantilever's inverse Q-factor. In SFA, friction is measured as the force necessary to shear a lubricating film squeezed between two step-free crystal surfaces. In QCM, a substrate is oscillated and the 2D solid adsorbate monolayer dissipates frictional energy while inertially sliding on its surface, and that reflects as the extra power necessary to keep a steady-state oscillation. It should be clear that besides these main atomistic type experiments there are of course many others, particularly amomg those whose character is more macroscopic, that would require more space and time, and whose immediate physical significance would be less transparent.

Yet another sort of experiments with a bearing on the physics of frictional sliding between two crystals has developed more recently in the wake of optical lattice systems. One-dimensional (1D) chains of cold atomic ions, or two-dimensional (2D) crystallized monolayers of charged colloids can be dragged to move across an optical lattice, thus constituting simplified but instructive emulators of the real sliding between two crystalline surfaces, here under well controlled conditions.

\section{Microscopic vs Macroscopic Friction Laws}

How do the overall results and understanding of these nano and meso-scale frictional experiments compare with the classic, centuries old laws of macroscopic friction? Not surprisingly, they are actually dif- 
ferent. In particular (i) the frictional force $\mathrm{F}$ is not independent of the area $A$ of contact, but grows instead roughly like $F \sim$ const $+A^{\gamma}$, were $\gamma$ is an exponent, usually not larger than 1 , that depends on the situation. In the macroscopic limit of large $A$, any exponent $\gamma<1$ will of course recover the macroscopic independence upon area; (ii) the frictional force $F$ is not proportional to the normal load $L$, but often grows as $F$ $\sim$ const $+\mu L$ where the additive constant accounts for adhesion: two adhering surfaces will experience friction even at zero load; in the macroscopic case, roughness and dirt practically eliminate adhesion; (iii) the (kinetic) friction force $F$ does depend on the sliding velocity $v$. Generally, in all cases of smooth, "easy" sliding, friction is "viscous", i.e., $F \sim v$. In "hard" dry sliding, conversely, where two surfaces touch in a small set of contacts, each microscopic contact advances by stopand-go events, so-called "stick-slips", which make friction grow very slowly, $F \sim \log v$, nearly independent of velocity. The (Coulomb's) law of macroscopic dry friction, $F$ independent of velocity, can therefore be argued to approximately reflect a myriad of microscopic contacts, each advancing by stick-slip, like a sort of marching caterpillar.

\section{Present Status of the Theory of Friction}

Macroscopic sliding friction is, from the point of view of physics, an emergent phenomenon. Every atomistic frictional event can, as it were, be described and reproduced by a suitable time-dependent calculation based on standard microscopic physics laws, classical or quantum. That is the route which theorists like ourselves are busy following nowadays for the study of nanofriction. It is however impossible, it actually makes no sense, to push this type of calculation to the macroscopic, multi-contact case. And not just because the computer budget would explode, which it would. In that limit it may rather be expected that, as for example is the case with circuit electronics or thermodynamics, some kind of emergent law should exist to describe mesoscopic/macroscopic friction, irrespective as it were of the underlying atomistic nitty-gritty. That is a honorable task for a theorist: and I in fact believe that we made some modest progress in that direction, which I shall mention near the end of this presentation. Nonetheless it is still fair to say that as of now an emergent description for friction does not yet exist, and its development stands as a challenge for the future. 
Given that starting point, we must face friction with what tools we have. Here is the list of available tools:

1. Linear response theory.

2. Simple nonlinear frictional models.

3. Classical non-equilibrium molecular dynamics simulations.

4. Markov State Model probabilistic approaches (work in progress).

\section{LineAr RESPONSE THEORY}

Sliding friction is a time-dependent, non-equilibrium phenomenon. Although generally violent, as in the case of stick-slip, there are frictional situations e.g., (a) in the smooth viscous sliding of diffusive molecule or cluster on a surface, or in noncontact AFM dissipation; or else (b) in the frictional loss felt by a fast, weakly coupled perturbing "slider" where the frictional contact is weak enough that linear response theory can still be applied.

Systems of type (a) can be described as weakly perturbed Brownian objects. If $D$ is the (2D) diffusion coefficient of the Brownian slider on the surface before the weak sliding force is applied, then the fluctuation-dissipation theorem applies, friction obeying Einstein's equation $D \eta=k_{B} T$. Once an infinitesimal sliding force $F$ is applied, the resulting friction is viscous, with sliding velocity $v=F / \eta$ and a dissipated frictional power

$$
W=F v=\eta^{-1} F^{2}=\left(D / k_{B} T\right) F^{2},
$$

an interesting result, showing that linear response dissipation is quadratic in the applied force, proportional to diffusion (itself temperatureactivated).

b) Systems type (b) consisting of a fast "slider" (actually more of a "flier") losing an infinitesimal part of its energy by a single weak "collision" with a surface. This kind of energy loss process is familiar in scattering theory, where the energy loss rate is well described by the Born's approximation,

$$
W=F v=\pi^{-1} \int_{0}^{\infty} v Q \operatorname{Im} \chi(Q, v Q)|V(Q)|^{2} d Q
$$

where $\chi Q, \omega$ is the free surface's linear response function, and $V(Q)$ the Fourier transformed slider-surface interaction potential. As with the Brownian slider, the frictional power is again quadratic in the slider's 
speed, and the temperature dependence is now embedded inside the response function.

These two linear response approaches describe totally different frictional regimes, and while both yield an effectively viscous friction force proportional to $v$, their temperature dependencies can clearly be different and even opposite. Both approaches can be applied with success and quantitative accuracy compared against explicit simulations and experiments where available. As an example, in Fig. 3 the averaged friction force $\langle F\rangle=\langle W\rangle / v$ calculated in the weak interaction limit for a smooth $1 \mathrm{D}$ sliding by the linear response formula above, shows an absolutely remarkable parameter-free agreement with an earlier purely numerical simulation.

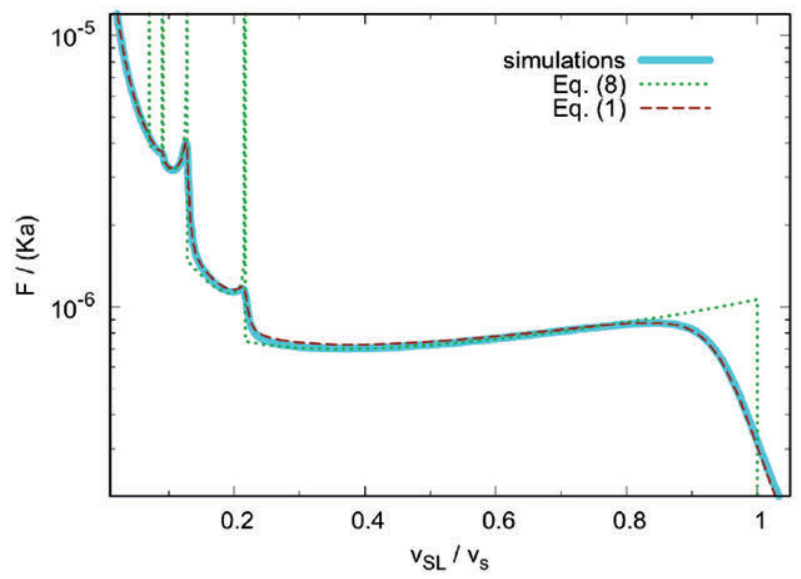

Fig. 3. Average friction force $\langle F\rangle=\langle W\rangle / v$ as a function of sliding velocity $v$ (here called $v_{S L}$ and normalized by the sound velocity) for the Prandtl-Tomlinson $1 D$ model of Fig. 4. Result obtained in the weak interaction limit where sliding is smooth and linear response type (b) can be applied. Friction peaks occur when the phonon group velocity coincides with the slider's $v$. Note the perfect agreement between linear response theory and earlier simulations.

From E. Panizon et al., Physical Review B 97, 104104 (2018).

\section{Simple Frictional Models}

As anticipated above, linear response theory only rarely applies to friction, generally involving violent nonlinear phenomena like stick-slip. They were historically rationalized by simple heuristic models. The socalled Prandtl-Tomlinson model, dating back to 1928, consists of a single 
classical point particle (the slider) of mass $M$ pulled by a spring $k$ with speed $v$ to move in a periodic potential of depth $V_{0}$ and spacing $a$. Depending on the dimensionless ratio $r=4 \pi^{2} V 0 / \mathrm{ka}^{2}$, which represents the ratio between the stiffnesses of the tip-substrate potential and the pulling spring $k$, this model describes smooth friction for $r<1$, and stick-slip friction for $r>1$. In its utter simplicity, it is still much used to represent for example AFM friction including both regimes, as sketched in Fig. 4.
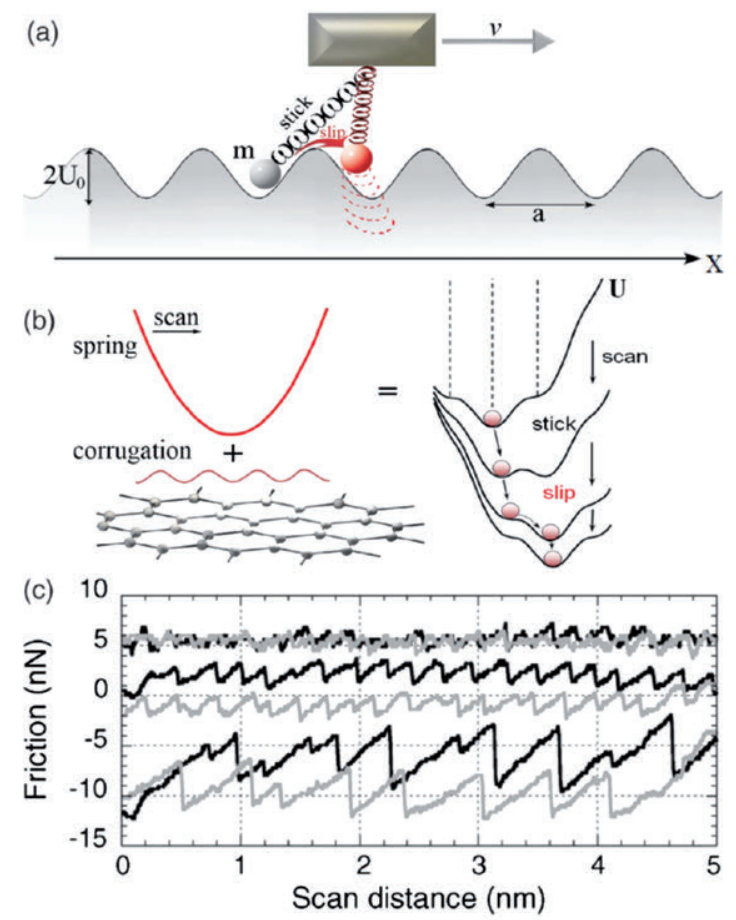

Fig. 4. (a) Cartoon of the Prandtl-Tomlinson model, (b) energy landscape for a soft spring (large $r$ ). The total potential (harmonic spring plus sinusoidal substrate) exhibits different metastable minima, giving rise to stick-slip; (c) representative AFM friction patterns, demonstrating transitions for increasing load from smooth sliding (top) to single (middle) and mostly double stick-slips (bottom). Very similar patterns can be generated within the Prandtl-Tomlinson model as function of increasing $r$. From A. Vanossi et al., Reviews of Modern Pbysics 85, 529 (2013).

The Prandtl-Tomlinson idea was further extended by the FrenkelKontorova model (1938), where the sliding particle is replaced by an infinite chain of particles with spacing $a_{c}$ connected by harmonic springs $\mathrm{K}$, 
thus representing the sliding of two crystals. (Fig. 5) Physically interesting situations occur in this model as a function of two parameters, the (inverse) interaction strength parameter $g=K / V_{0}$, and the (in)commensurability parameter $a_{d} / a$. When $a_{d} / a=1$, or any rational number, the two crystals are commensurate, locked together at a minimum of total energy and effectively pinned, so that a nonzero force $F_{s}$, the so-called static friction, is required to initiate the frictional sliding. Conversely, in the incommensurate case, where $a_{d} / a$ is irrational, the total energy is a constant, independent of the relative position of the two crystals. One would then logically surmise in that case (and it was widely believed until relatively recently) that two incommensurate crystal surfaces should slide freely, and their static friction would vanish. In 1983, Aubry proved mathematically that it is not always so. Sliding is only free above a critical inverse interaction strength $g_{c}$, a quantity dependent on $a_{c} / a$. At $g=g_{c}$ a dynamical, continuous phase transition occurs, below which ergodicity is broken, giving rise to pinning and finite static friction despite incommensurability, as sketched in Fig. 6. This is easiest to understand in the limit where the interparticle spring $K=g V_{0} \rightarrow 0$ becomes infinitely slack, whereby each particle is free to move to the closest minimum of the periodic potential. In that case the occupation probability of any potential maximum or its neighborhoods falls mathematically to zero (at zero temperature), and the chain as a whole cannot shift bodily in response to an infinitesimal shearing force.

THE AUBRY TRANSITION

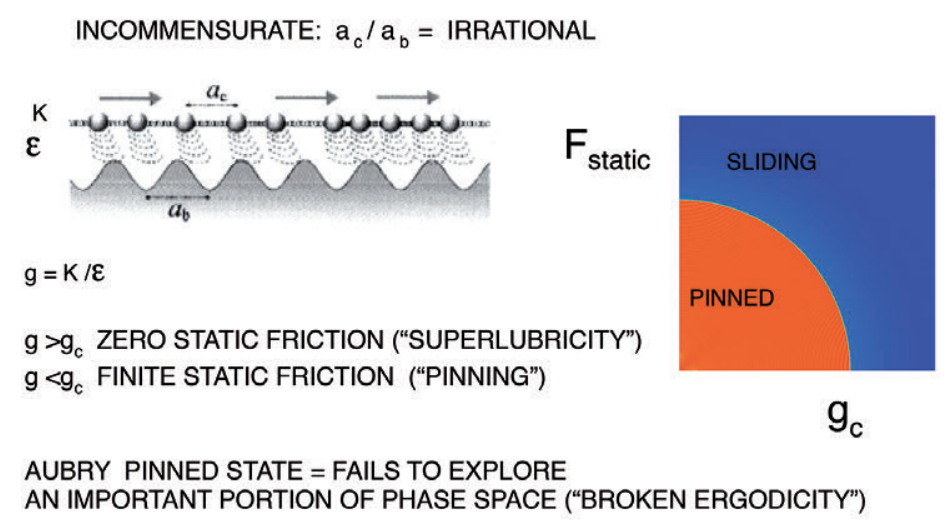

Fig. 5. The Frenkel-Kontorova model and the Aubry transition, where static friction goes from zero in the free sliding case $g>g_{c}$, to finite in the pinned case $g<g_{c}$. 
The Aubry transition has been emulated experimentally in 1D cold ion chains, and in 2D colloid monolayers, both immersed in incommensurate optical lattice potentials. The $1 \mathrm{D}$ result was found to follow closely the continuous pinning transition predicted in the Frenkel Kontorova model. The 2D transition, for which no analytical theory is available, was found instead to be of first order, as predicted by earlier molecular dynamics simulations. (Fig. 6).
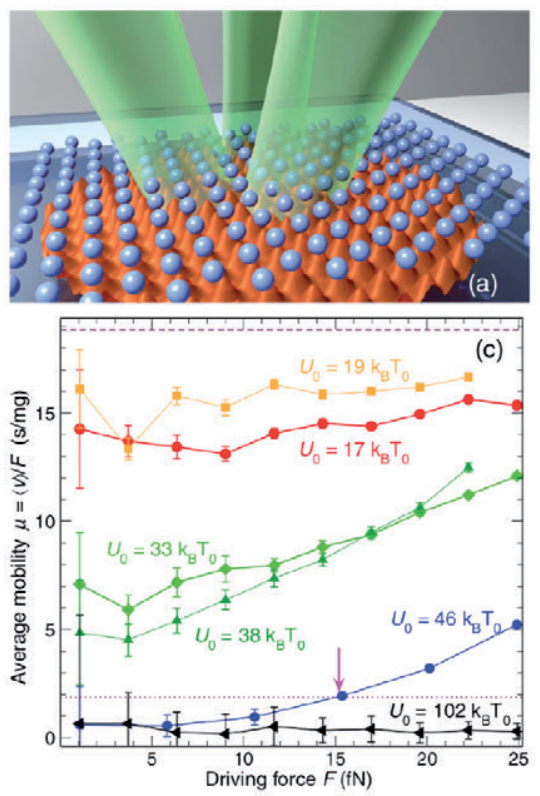

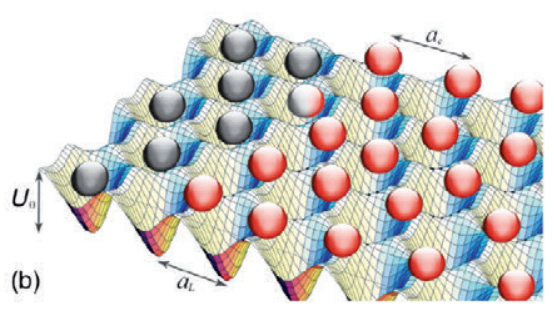

Corrugation amplitude $U_{0} / U_{c}$

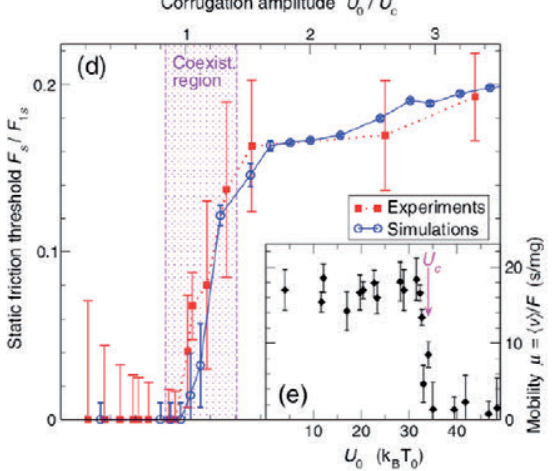

Fig. 6. Driving a colloidal monolayer across a periodic laser potential.

(a) Schematic view of an experimental setup. (b) Corresponding simulated model. (c) Measured mobility of an incommensurate $\left(a_{d} / a=1.19\right)$ colloid monolayer vs driving

force for different corrugation amplitudes $V_{0}$ in the free sliding (red and orange symbols), coexistence (green symbols), and pinned phase (blue and black symbols).

The dashed line shows the maximum mobility, while the dotted line shows the minimum threshold mobility to detect sliding. The pink arrow points at the crossing of this threshold mobility, defining the value of $F_{s}$ for this value of $V_{0}$.

(d) Static friction force $F_{s} v s V_{0}$ obtained from experiments (solid symbols) and simulations (open symbols); the shaded area shows the coexistence region, across which Aubry transition takes place. (e) The monolayer mobility under the action of the smallest experimentally accessible driving force $F_{\text {min }} \simeq 1 \mathrm{fN}$; the critical corrugation is defined by the sharp drop in the mobility (arrow).

From T. Brazda et al., Physical Review X 8, 011050 (2018). 


\section{Molecular Dynamics Simulations (Non-equilibrium)}

After linear response and the nonlinear frictional models, direct non-equilibrium molecular dynamics (MD) computer simulations represents the main theoretical instrument. Of course, there are issues concerning size $N$, velocity $v$ and time scale, necessarily much smaller in simulations $(N<1$ million particles, $v>0.1 \mathrm{~m} / \mathrm{s}, \tau<1 \mu \mathrm{s})$ than in a frictional experiment $\left(N>10^{20}\right.$ particles, $\left.v \sim 1 \mu \mathrm{m} / \mathrm{s}, \tau>>1 \mathrm{~s}\right)$. With luck, the active region in nanoscale friction is not that large; other issues too are in that case usually resolved by suitable extrapolations. (Fig. 7) MD simulations thus provide unique insight in nanofriction, sometimes overturning conventional wisdom. The classical dynamics of all atoms (appropriate for the descrIption of a solid at sufficient temperatures $T$ $>0.2 T_{D}$ where $T_{D}$ is the Debye temperature) is obtained by numerically solving Newton's (or Langevin's) equations of motion based on suitable interparticle interaction potentials and the corresponding interatomic forces. The geometry of the sliding interface and the boundary conditions can be chosen to explore friction, adhesion, and wear. A thermostat, or other form of damping, must be introduced in order to eliminate the Joule heat and obtain a frictional steady state. After specifying the initial coordinates and velocities of the particles, the classical differential equations of motion are integrated numerically.

(b) FRICTIONAL SIMULATIONS: CLASSICAL NON-EQUIL. MOLECULAR DYNAMICS

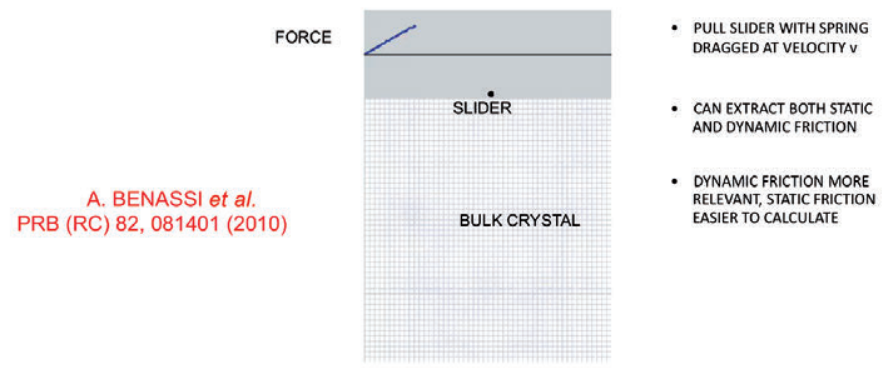

- MODELING DESIRED PHYSICS, SELECT FORCE FIELD, SELECT TIP MODEL

- REALISTIC ELIMINATION OF JOULE HEAT NEEDED

- TIME LIMITATIONS -- CAN LEARN EVEN WITH LARGE SPEEDS

- SIZE LIMITATIONS - SMALL SIZES, OK FOR NANOFRICTION

- HIGH TEMPERATURE ASSUMED - NEGLECT QUANTUM EFFECTS

Fig. 7. Illustrating a setup for non-equlibrium MD simulation of friction by e.g., a point-like slider on a crystal surface. 
The literature provides countless realizations of MD simulated nanofriction. As an example, we show in Fig. 8 the simulated friction obtained in an interesting case describing a (point-like) "tip" sliding over the (1D) surface of a 2D crystal model, where the model contained in this case a bulk phase transition at a critical temperature $T_{c}$. The simulated friction reveals a broad peak accompanying the bulk critical state, reflecting the increased dissipation accompanying the large (in principle divergent) spatial extension of the slider's influence at the phase transition point.

PREDICTED AFM FRICTION ANOMALY AT BULK PHASE TRANSITION

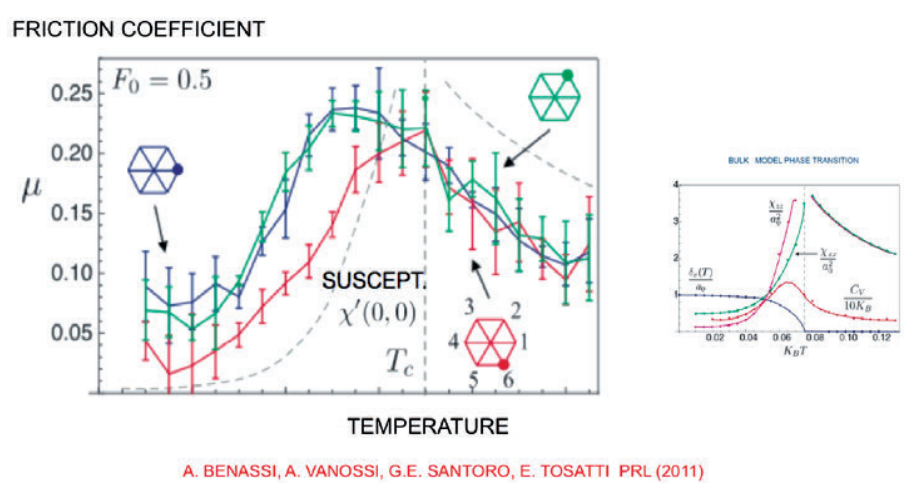

Fig. 8. The friction felt at the surface of a crystal which undergoes a bulk phase transition is found by MD simulation to exhibit an anomaly whose detail depends on the bulk order parameter below $T_{c}$. The peak temperature shift relative to the bulk $T_{c}$ is a finite-size effect.

From A. Benassi et al., Physical Review Letters 106, 256102 (2011).

This result exemplifies the concept that nanofriction can serve as a "spectroscopy for the blind". Physical phenomena taking place inside a solid, such as a bulk phase transition, can be picked up by a non-invasive tip feeling the nanofriction at a surface - a sort of "Braille spectroscopy".

Besides the friction force required to initiate and maintain the sliding of a body or a tip, another frictional quantity of physical interest is the "phoretic" force felt by a body or a tip in contact with a solid where a current, for example electronic or thermal, is circulating. Electromigration MD simulations, necessarily ab initio because electrons are quantum, are not yet available for the electrical case. 
Conversely we recently gave a first description of the phoretic force generated by a thermal current. The MD simulation of a gold nanocluster physisorbed on freestanding graphene and transported by a temperature gradient in the graphene sheet gave a surprising result. The thermophoretic force is, at least for distances below one or two hundred nanometers, not proportional to the thermal gradient $\operatorname{grad} T$ as expected by the macroscopic Soret law, but rather proportional to the crude left-right temperature difference $\Delta T$. The underlying physics is that flexural phonons in graphene, phonons which by kicking the adsorbed cluster act as carriers of the phoretic force, propagate ballistically rather than diffusively over the first few hundred nanometers. Before the onset of diffusive scattering, ballistic phonons flowing from hot to cold are conserved, and so is the force with which they kick the adsorbed cluster (Fig. 9).

SIMULATION: GOLD CLUSTER ON SUSPENDED GRAPHENE

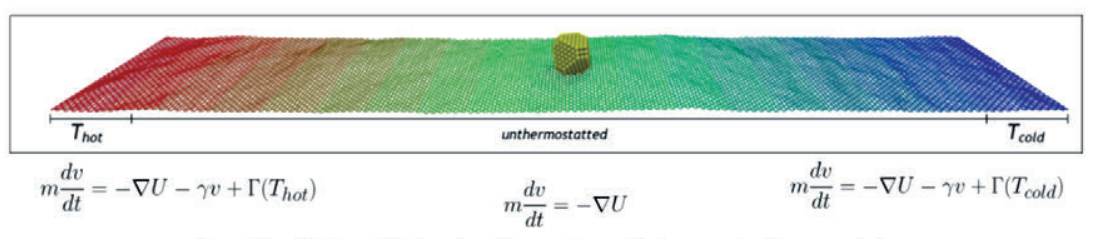

Non-Equilibrium Molecular Dynamics with Langevin thermostats

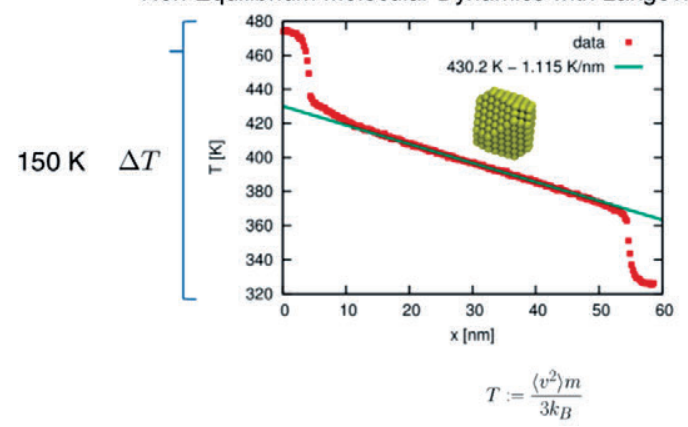

Fig. 9. MD simulation predicting and understanding the thermophoresis of a physisorbed cluster (or molecule) on a membrane-like substrate like graphene. Details in E. Panizon et al., Proceedings of the National Academy of Sciences (US) 114, E7035 (2017).

This work also exemplifies the capability - long claimed in fact of realistic MD simulations and associated theory to predict, and in lucky cases to discover, as it were - new results ahead of experimental 
investigations, not yet realized as in this case. We look forward to being proven wrong or hopefully right in this specific case.

\section{Markov State Model Theory}

Sliding friction can, as mentioned, be measured, modeled, simulated but, disappointingly, not properly formulated and described theoretically. In the purely classical sliding of a body on another, for example, there is no unprejudiced way of identifying and determining a handful of variables (as opposed to the $10^{23}$ atomic coordinates and velocities) that obey their own well defined equations of motion, describing the essence of the frictional process. The case of nanofriction, where atomistic simulations are sometimes possible as I just showed, makes if anything this theoretical vacuum even more insulting. One clue to the problem which we are following is the observation that the important frictional events, especially those of mesoscopic and macroscopic character, generally involve large scale, slowly evolving variables, whereas a plethora of other fast variables only merit to be integrated upon, if only we knew how. A predictable result of that integration would be a certain loss of memory between one slow event and the next, suggesting in the extreme limit a Markovian process. In a recent attempt, we therefore proposed that Markov state modeling (MSM) — a probabilistic approach commonly applied in biological systems to characterize the coarse-grained kinetics of systems characterized by an equilibrium measure - could be extended to the strongly non-equilibrium, non-linear problem of sliding friction. A first attack was demonstrated in a simple 1D toy model, a 10-atom Frenkel Kontorova model where, despite the difficulty represented by a timegrowing phase space, non-equilibrium MSM was shown to describe adequately the forced dynamics of steady-state sliding friction. A long steady-state frictional simulation first, then the choice of a metric, then the clustering in phase space, are the starting ingredients of this approach. The probabilistic analysis of phase space evolution leads to the recognition of Markovian evolution, and to the building of a timedependent transition matrix. Diagonalization thus leads to the analytical identification of a few slowest eigenvalues and corresponding collective variables ("excitations") describing the main events occurring in the course of steady-state sliding (Fig. 10). 


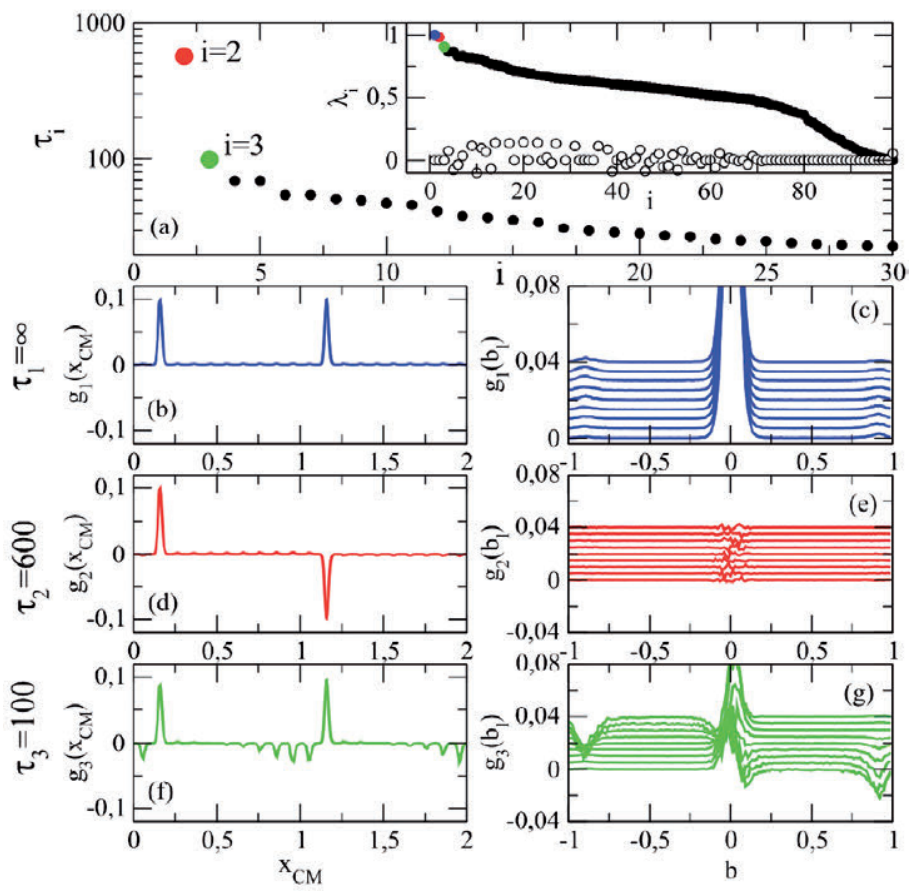

Fig. 10. Markov State Model description of a1 0-particle commensurate Frenkel Kontorova model. (a) Characteristic eigenvalues and time scales (in the inset, imaginary part in white) of the Markov Transition Matrix (averaged over 10 realizations with $N=105$ each). (b), (d), and ( $f$ Center-of-mass steady state probability distribution and perturbations predicted by the first three eigenvectors. (c), (e), and (g): these same functions for the bonds bl, gi (bl) (spaced vertically for clarity). From F. Pellegrini et al., Physical Review E 94, 053001 (2016).

That construction, in essence, provides an unprejudiced analytical model of the slowest time evolution of probabilities. This kind of approach, presently still under development, represents in our view a first step towards a theory of friction and a methodological advance of significant importance.

\section{CONCLUSIONS}

Nanofriction is driving a renaissance of sliding friction and mechanical dissipation as a physical phenomenon. In this very short 
and biased tour I attempted to describe some of our own modest attempts at making sense of that evolution it from the observation angle of a condensed matter theorist. Much more is going on than what I presented. Yet, I hope that a certain feeling of where we stand, and especially of what is within reach and what is not, could be obtained.

In addition to my brave collaborators, listed in first page, I wish to express heartfelt thanks to Professors Attilio Rigamonti and Andrey Varlamov for inviting me to join this exciting occasion and present this contribution, to Dr. Adele Bianchi Robbiati for coaxing me ever so gently into writing it up in good time, and to the Accademia Istituto Lombardo for the honor of membership bestowed upon me six years ago. 